\title{
Blended foods for tube-fed children: a safe and realistic option? A rapid review of the evidence
}

\author{
Jane Coad, ${ }^{1}$ Alex Toft, ${ }^{1}$ Susie Lapwood, ${ }^{2}$ Joseph Manning, ${ }^{3}$ Mark Hunter, ${ }^{4}$ \\ Huw Jenkins, ${ }^{5}$ Clare Sadlier, ${ }^{5}$ Julie Hammonds, ${ }^{6}$ Ailsa Kennedy, ${ }^{7}$ Simon Murch, ${ }^{8}$ \\ David Widdas ${ }^{9}$
}

- Additional material is published online only. To view please visit the journal online (http://dx.doi.org/10.1136/ archdischild-2016-311030).

For numbered affiliations see end of article.

\section{Correspondence to} Professor Jane Coad, Faculty of Health and Life Sciences, Children and Families Research (CFR), Centre for Technology Enabled Health Research (CTEHR), Coventry University, Security House, Mile Lane, Coventry CV1 2NL, UK; aa9737@coventry.ac.uk

Received 13 April 2016 Revised 10 September 2016 Accepted 18 September 2016 Published Online First 18 October 2016

\section{ABSTRACT}

With the growing number of children and young people with complex care needs or life-limiting conditions, alternative routes for nutrition have been established (such as gastrostomy feeding). The conditions of children and young people who require such feeding are diverse but could relate to problems with swallowing (dysphagia), digestive disorders or neurological/muscular disorders. However, the use of a blended diet as an alternative to prescribed formula feeds for children fed via a gastrostomy is a contentious issue for clinicians and researchers. From a rapid review of the literature, we identify that current evidence falls into three categories: (1) those who feel that the use of a blended diet is unsafe and substandard; (2) those who see benefits of such a diet as an alternative in particular circumstances (eg, to reduce constipation) and (3) those who see merit in the blended diet but are cautious to proclaim potential benefits due to the lack of clinical research. There may be some benefits to using blended diets, although concerns around safety, nutrition and practical issues remain.

\section{INTRODUCTION}

This article presents the findings from a rapid review of the available peer-reviewed literature which considers a blended $\operatorname{diet}^{\mathrm{i}}$ for children as an alternative to standardised commercial feeds. This is against the backdrop of increasing anecdotal evidence that families are choosing such a diet for their children and finding it beneficial. Health professionals' responses however have been mixed. Most notably the statements from the British Dietetic Association who does not endorse the practice of using blended diets, although guidance has been issued $\mathrm{d}^{\mathrm{ii}}$ and some manufacturers of gastrostomy buttons, such as Vygon, have stated that their equipment can be used for blended diet. ${ }^{1}$

Medical practitioners and dietitians support the use of commercial formula feeds as they contain the precise measures of the calories, macronutrients and micronutrients that the child requires. ${ }^{2}$

'We will use the term 'blended diet' throughout to refer to the use of blended food administered through an enteral tube. This food contains, at least in part, normal solid food or dairy products usually prepared in the home, which is blended to produce a fine puree or liquid and used through the enteral tube. Alternative phrases such as blenderised, liquidised and homebrew have been used.

${ }^{i i}$ Seehttps://www.bda.uk.com/improvinghealth/health professionals/liquidised_feeds.

\section{What is already known on this topic?}

- Current national guidelines call for the use of commercial formula to be used over blended food wherever possible.

- There are risks which should be kept in mind when considering the use of a blended diet, particularly with regard to infection control.

- The desire to nurture and feed is taken away from parents whose children are fed via gastrostomy.

\section{What this study adds?}

- There is some evidence of benefits as a blended diet could improve some symptoms of diarrhoea and/or vomiting and could give families control of the development of their child.

- Recent research has suggested that there are concerns over the suitability of blended diet when using new tube equipment and that such diets are nutritionally variable and imprecise.

- Further high quality, empirical studies are required to understand the benefits, impact and outcomes of blended diets and the experiences of the children and families who use them.

Subsequently these feeds are seen to be easily quantifiable, convenient, portable, pasteurised and balanced. Families using nutritional feeds are provided with packaged feeds, a specific time schedule and a prescribed number of ounces per feeding. However, many families have reported that this approach focuses only on the medical nature of nutrition and increases the separation of the child from the family meal and the feeding relationship that families value. ${ }^{2}$

Although the use of blended diets via gastrostomy are receiving growing support in international literature as an alternative to nutritional formula feeds, their effects have not been well studied or evaluated in the UK. There is an urgent need to address this issue to overcome the risk of families trialling the diet for themselves in the absence of any professional guidance or literature. Importantly, families who have been informed about the potential benefits of a blended diet approach have helped to 
generate and drive interest, making the push to recognise blended diets user-led. It is often the issue of choice that families hold on to, as they chose how to feed their own child. It is hoped that this literature review will highlight key considerations of a blended diet to carers, dieticians and family members.

\section{AIMS}

The overall aim of this paper will be to review the literature in order to explore research opinion on whether blended diet is a valid alternative to commercial formula for children who are enterally fed via a gastrostomy. There were no limitations put on how this was qualified and included articles that explored and reported medical, nutritional and social outcomes and experiences.

\section{METHOD}

A rapid review method was employed using systematic principles. These will be detailed throughout the following section.

The online supplementary table summarises the studies which were included in the review. Figure 1 highlights the methodological approach and the inclusion and exclusion criteria in greater detail.

\section{Search strategy}

See online supplementary appendix A for protocol.

Key databases were searched including PubMed, MEDLINE, CINAHL, PsychINFO and Google Scholar. Searches took place between August and December 2014 and detailed searches were saved. The searches and extractions were verified by additional reviewers.

\section{Inclusion/exclusion criteria}

Results included peer-reviewed journals only where research outcomes are presented.

Inclusion criteria:

- Studies need to be published in English due to the time constraints of the review.

- Studies had to explore a blended diet as a real alternative to commercial formula feeds.

- Studies that examined blended diets and gastrostomy care for adults were included if findings were easily transferable to children and young people ${ }^{\mathrm{iii}}$ with gastrostomies.

- For inclusion, studies need to focus on gastrostomy and must consider blended diets as a type of feed. This is important because although there are few studies specifically exploring blended diets, there are studies which consider a blended diet while examining commercial formulas.

Studies which did not focus on original research (eg, literature reviews) were excluded as were articles which were solely theoretical in nature (eg, did not report outcomes).

\section{Data extraction and critical appraisal}

A data extraction sheet was used to record the critical appraisal of the selected studies. Data extracted included topic, study design, methodology, outcomes and author's conclusions, limitations and reasons for exclusion, if applicable. Throughout the process checking and verification took place by at least an additional two members of the team.

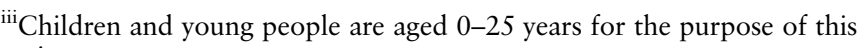
review.

\section{Reporting}

Information from the dataset was synthesised into a coherent narrative to address the aims and objectives of the review. Themes were extracted from the data pertaining to experiences and perspectives around the use of blended diets by children and young people with a gastrostomy. These themes were synthesised into a narrative presentation. Although studies often use phrases such as tube feeding and enteral feeding, and this is reflected in reporting, we focus on feeding via gastrostomy as this is more practically viable.

\section{RESULTS}

Eighteen articles were included in the review and are summarised in the online supplementary table. Through synthesis of the themes, this review explores the current literature on blended diet with regard to the range of conditions that blended diet is used for, the nutritional value of a blended diet, the impact of a blended diet on family and issues of choice, difficulties and risks associated with home-made blended diets and the benefits of home-made blended diets against the shortcomings of formula feeds.

\section{Range of conditions}

Pentuik et $a l^{3}$ explored the possible benefits of a blended diet for children after fundoplication surgery. It was hypothesised that a blended diet may reduce gagging and retching which have been associated with using commercial formula. Another study which focused on a specific condition was a Croatian study conducted by Kolacek et al. ${ }^{4}$ The authors tested whether a modular diet would improve chronic diarrhoea in infants aged $<1$ year.

Other studies have explored a wider population of those who require enteral feeding. The study of Daveluy et $a l^{5}$ included those with digestive disorders, neurological and muscular disorders and malignancy. A Polish study conducted by Klek et $a l^{6}$ included a significant population of young people ( 39 were aged $\leq 17$ years) and included a sample of those with neuromuscular swallowing disorders, cancer-related dysphagia and cystic fibrosis.

\section{Nutritional value of blended diets}

In general, studies did not specify the recipes of blended diets beyond stating it was home-cooked food. Others studies involved recipes tailored to the child's health and the family's traditional foods. ${ }^{2}$ However, actual recipes were documented and tested by two studies. First, Kolacek et $\mathrm{al}^{4}$ tested a modular diet comprising boiled mince chicken meat, sunflower oil emulsion, sucrose and cornflower and then compared this with Pregomin formula. Borghi et $a l^{7}$ tested several recipes:

1. Beef/chicken with legumes

2. As above, with cooked beans, green leaves and eggs

3. As above, with cow's milk and oils (vegetable, soya bean, corn)

Pentuik et $a l^{3}$ provided a sample menu which could be modified; this included strained meats, plums/bananas, strained pears/ applesauce, strained squash/sweet potations, infant cereals, oil, yoghurt, corn-starch/sugar and formula/milk. This resulted in $25 \mathrm{oz}$ providing 942 calories.

The nutrient composition of feeds prepared from normal foodstuffs depends on the nutrient compositions of the foods used. $^{8}$ As a result, such feeds are variable in their content. Furthermore, these compositions can vary according to the geographical source of the food, the season and stage of maturity when the food was harvested, food-processing methods, storage conditions and cooking methods. Schuitema ${ }^{9}$ argued that it is 
Figure 1 Flow diagram showing the results of the searches.

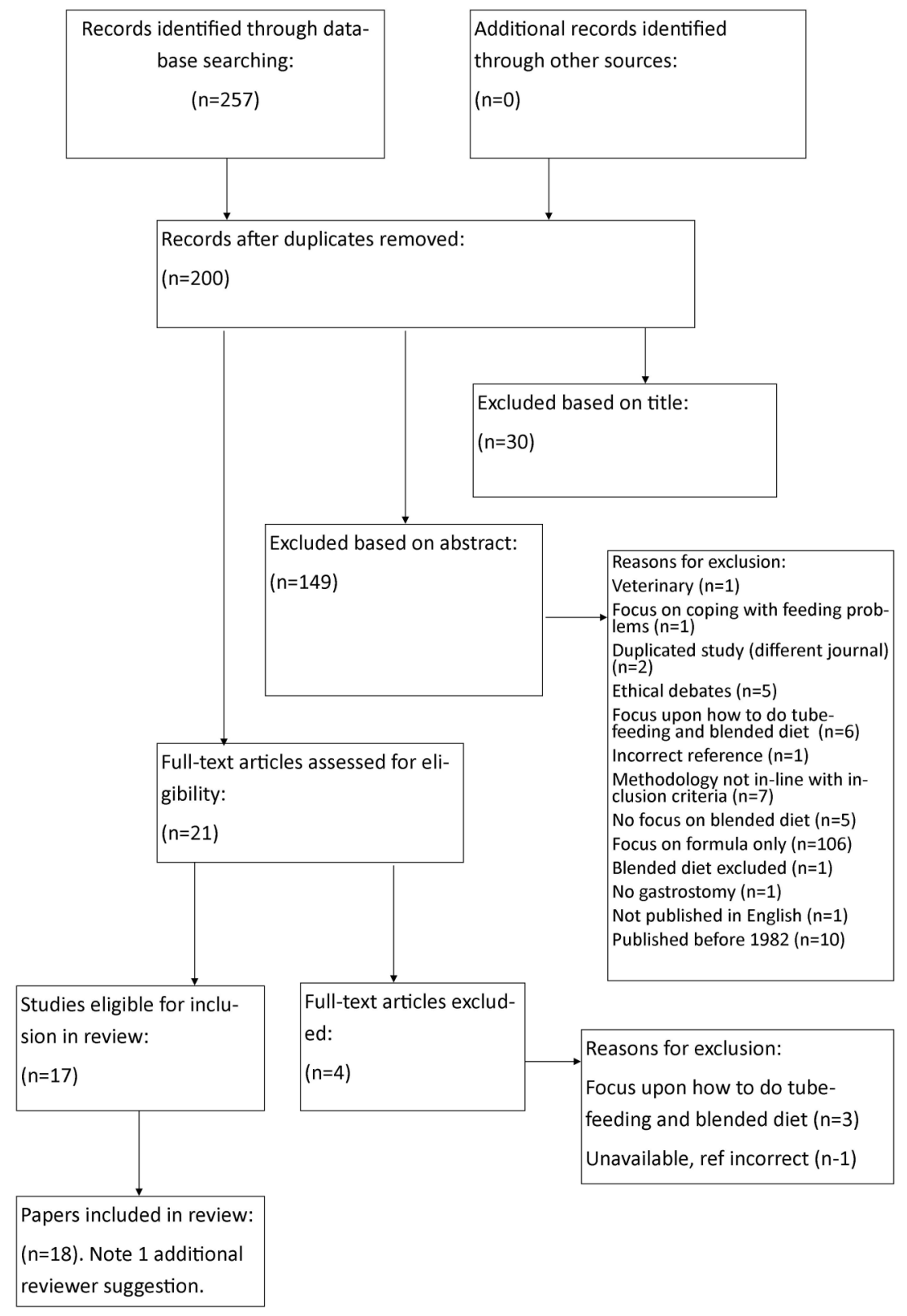

difficult to meet a patient's nutritional needs using a home-made liquid diet since it will have a much lower calorie density than prescribed feeds and require much larger volume. Older studies ${ }^{10}$ also highlighted the inaccuracy of the calorie count for blended food. Novak et $a l^{2}$ also add that, when families attempt to produce blended diets on their own, the result is quite often inadequate fluid, protein and nutrient intake for the child. It is recommended that with children who have high energy needs such as those with hypertonia, a high calorie formula could be added to their blended feed recipe. Schuitema ${ }^{9}$ suggests that patients' intake of blenderised diets should be carefully monitored along with any outputs (eg, urine; faeces), weight changes and symptoms. Recent research has suggested however that variation in diet is the key to a healthy gut. Claesson et $a l^{11}$ have suggested that the reliance on a single formula may lead to an impoverishment of microbial diversity in the enteric flora, although a formula feed is theoretically nutritionally adequate. It is concluded that use of a single formula could contribute to long-term ill-health in the elderly.
Although not exploring the blended diet as such, research has also suggested that commercial formula has links to obesity. Use of blended diet may be more advantageous in this respect. ${ }^{12}$

\section{Family impact and choice}

Families reported that preparing blended diets gives them more control in their children's growth and feeding and allows them to nurture children with a gastrostomy with food that they would give to orally fed children. ${ }^{2}$

Novak et $a l^{2}$ suggests that paediatricians should support and encourage families to use blenderised feeds for their children, although this should be guided by professionals. Families question whether one formula, one diet or one recipe could provide all the nutrition needed to maximise health and growth for children with gastrostomy. ${ }^{13}$ This sense of empowerment and taking ownership of care is important to consider with regard to gastrostomy feeding as there is a strong instinct to nurture and to feed children. ${ }^{14}$ Although studies have not been conducted on the blended diet, research has shown that 
gastrostomy feeding leads parents to feel like they are no longer nurturing their child. ${ }^{15}$

\section{Difficulties and risks associated with home-made blended diets}

Studies have highlighted the persistent and negative symptoms associated with the continuous use of commercial nutritional feeds including constipation, diarrhoea, reflux, retching and gut failure associated with both the child and the family's quality of life. ${ }^{16}$ However, clinical risks associated to the use of blended diets have also been identified in the literature. Importantly, current National Institute for Health and Care Excellence guidelines state that, wherever possible, pre-packaged commercial formulas should be used over any enteral feeds that have to be prepared. Such preparation has risk of infection and contamination. $^{17}$

The inside diameter of fine bore tubes is small $(2-3 \mathrm{~mm})$ and they can easily block because of coagulation of proteins and minerals. ${ }^{18}$ Feeds, therefore, need to be non-viscous and free of particles that could block the tube. However, the mean viscosity of blended feeds prepared for the 21 samples in the study by Sullivan $e t a l^{8}$ was reported to be more than 43 times higher than typical commercial formulas. Consequently, it was felt that some of these samples would not flow easily through nasogastric or nasoenteric feeding tubes and could occlude these tubes. To prevent tube occlusion from a high-viscosity formula, rapid feeding by bolus method or the use of large bore feeding tubes was recommended. Sullivan et $a l^{8}$ concluded that these methods of feeding were poorly tolerated compared with continuous feeding through a small bore feeding tube. Recent research conducted by Mundi et $a l^{19}$ has found that home-made blenderised recipes require more force to push through when using the new ENFit adapter which could have implications for using a blended diet in malnourished patients.

When a child starts receiving a blended diet, a food intolerance is often unmasked. It is recommended that a child should have had a period of gastrointestinal stability and absence of other major health changes before the blended diet is introduced. ${ }^{2}$

Schuitema ${ }^{9}$ presents a comprehensive list of rules and recommendations to minimise risks when using a blenderised liquid food diet. The list includes the avoidance of mixing additional fibres with liquid diets so that the mixture does not thicken and clog tubes. It is also recommended that in order to prevent bacterial contamination the real food for liquid diets should be pasteurised. Tubes should be rinsed every 4 hours and home-made feeds should always be enterally administered.

Additionally, Novak et $a l^{2}$ presents essential criteria to help to determine when a blended real food diet can be safely and competently used. This consists of the following points: when child is medically stable and has treated reflux; when appropriate weight or caloric intake is obtained; when motivated care providers with appropriate kitchen facilities are available; when a gastrostomy tube is at least $14 \mathrm{Fr}$ and when the gastrostomy site is well healed with no infection.

\section{Benefits of home-made blended diets and shortcomings of formulas}

Although outside of our inclusion criteria, a recent study has indicated that healthcare professionals report positive experiences when using a blended diet, ${ }^{20}$ and there is some evidence highlighting clinical improvements from a blended diet in an American study. ${ }^{3}$ Here a total of 33 children with mean age of 34 months were given a pureed by gastrostomy tube diet in an attempt to improve symptoms, nutrition and hydration. Findings showed that $73 \%$ had at least a 50\% reduction in symptoms after 2-6 months, no child had worse symptoms and over $50 \%$ had increased enteral intake. Similarly, Novak et $a l^{2}$ reported a greater volume tolerance and improvements in reflux and constipation when switching from commercial formula to blenderised tube feeding.

If a child has a medical plan allowing him or her to eventually undergo a transition from tube feeding to oral feeding, then blended diets are also thought to facilitate this process by introducing meal planning and by priming the gastrointestinal system. $^{2}$

\section{CONCLUSION}

The limited evidence suggests that a blended diet via gastrostomy might be effective for improving the food intake of those with chronic diarrhoea and those after fundoplication surgery. There is emerging evidence that a blended diet is being used by families who feel it is more effective than formula feeding, yet the evidence base is not established. There is a suggestion however that a blended diet has a wider social benefit, improving the relationship between child and parent and allowing families to become involved with tube feeding.

This review will help to provide vital guidance for families and professionals in knowing the benefits and risks of using real food blended diets via gastrostomy as an alternative to formula feeds. Findings can also be used to inform policy, practice and further research into safely trialling and evaluating the blended diet with gastrostomy-fed children and young people and their families. There is also potential for longer-term benefits from this review that extend beyond those that are reported, which include empowering families to be together for family mealtimes and improve food-sharing which families value so much.

More robust research is now needed to explore the risks, benefits, impact and outcomes of blended diets and the experiences of the children and families who use them. The use of blended diet via gastrostomy is becoming increasingly popular. A stronger evidence base would inform professionals as they advise families and would inform development of standardised guidance to support safe, evidence-based practice.

\section{Author affiliations \\ ${ }^{1}$ Children and Families Research (CFR), Centre for Technology Enabled Health Research (CTEHR), Coventry University, Coventry, UK \\ ${ }^{2}$ Helen and Douglas House, Oxford, UK \\ ${ }^{3}$ The University of Nottingham/Nottingham Children's Hospital, Nottingham, UK \\ ${ }^{4}$ Acorns Children's Hospices, Birmingham, UK \\ ${ }^{5}$ Department of Child Health, University Hospital of Wales, Cardiff, UK \\ ${ }^{6}$ Hope House Children's Hospices, Morda, UK \\ ${ }^{7}$ Liverpool Community Health, Liverpool, UK \\ ${ }^{8}$ Warwick University Hospital, Coventry, UK \\ ${ }^{9}$ South Warwickshire NHS Foundation Trust, Warwick, UK}

Twitter Follow Jane Coad @CovUni_CTEHR

Contributors JC: principal investigator; JC and AT: substantial contribution to conception, design, acquisition, analysis and interpretation of the data; drafting and revision of the work; final approval was given. JC, SL, JM, MH, CS, HJ, JH, AK, SM and DW: substantial contribution to conception and design of the data and are accountable for all aspects of the work.

Funding South Warwickshire Foundation Trust.

Competing interests None declared.

Provenance and peer review Not commissioned; externally peer reviewed.

\section{REFERENCES}

1 British Dietetics Association. BDA Policy Statement. Use of Liquidised Food with Enteral Feeding Tubes. 2013. https://www.bda.uk.com/improvinghealth/health professionals/policystatement_liquidisedfood (accessed Jul 2016). 
2 Novak P, Wilson KE, Ausderau K, et al. The use of blenderized tube feedings. Infant Child Adolesc Nutr 2009;1:21-3.

3 Pentuik S, O'Flaherty T, Santoro K, et al. Pureed by gastrostomy tube diet improves gagging and retching in children with fundoplication. JPEN J Parenter Enteral Nutr 2011;35:375.

4 Kolacek S, Grgurić J, Percl M, et al. Home-made modular diet versus semi-elemental formula in the treatment of chronic diarrhoea of infancy: a prospective randomized trial. Eur J Pediatr 1996;155:997-1001.

5 Daveluy W, Guimber D, Mention K, et al. Home enteral nutrition in children: an 11 year experience with 416 patients. Clin Nutr 2004;24:48-54.

6 Klek S, Szybinski P, Sierzega M, et al. Commercial enteral formulas and nutrition support teams improve the outcome of home enteral tube feeding. JPEN J Parenter Enteral Nutr 2011;35:380-5.

7 Borghi R, Araujo TD, Airoldi Vieira RI, et al. ILSI Task Force on enteral nutrition; estimated composition and costs of blenderized diets. Nutr Hosp 2013;28:2033-8.

8 Sullivan MM, Sorreda-Esguerra P, Platon MB, et al. Nutritional analysis of blenderized enteral diets in the Philippines. Asia Pac J Clin Nutr 2004;13:385-91.

9 Schuitema CFJ. Basics in clinical nutrition: diets for enteral nutrition home-made diets. J Clin Nutr Metab 2009;4:e168-9.

10 Gallagher-Allred CR. Comparison of institutionally and commercially prepared formulas. Nutr Support Serv 1983;3:32-4.

11 Claesson MJ, Jeffery IB, Conde $\mathrm{S}$, et al. Gut microbiota composition correlates with diet and health in the elderly. Nature 2012;488:178-84.
12 Sullivan PB, Alder N, Bachlet AME, et al. Gastrostomy Feeding in Cerebral Palsy: too much of a good thing? Dev Med Child Neurol 2006;48:877-82.

13 O' Gorman EA. Complete tube feeding: everything you need to know about tubefeeding, tube nutrition and blended diets. London: CreateSpace, 2012

14 Lambert BR, Han WM. Feeding and dietetic assessment and management. In: Sullivan $\mathrm{P}$, ed. Feeding and nutrition in children with neurodevelopmental disability. London: Mac Keith Press, 2009:57-85.

15 Sleigh G. Mothers' voice: a qualitative study on feeding children with cerebral palsy. Child Care Health Dev 2005;31:373-83.

16 Nicholson FB, Korman MG, Richardson MA. Percutaneous endoscopic gastrostomy: a review of indications, complications and outcome. J Gastroenterol Hepatol 2000;15:21-5.

17 NICE. Healthcare-associated infections: prevention and control in primary and community care. 2012. https://www.nice.org.uk/Guidance/cg139 (accessed Jul 2016)

18 Keighley MR, Mogg B, Bentley S, et al. "Home brew" compared with commercial preparation for enteral feeding. Br Med J (Clin Res Ed) 1982;284:163. PMCID: PMC1495566.

19 Mundi MS, Epp L, Hurt RT. Increased force required with proposed standardized enteral feed connector in blenderized tube feeding. Nutr Clin Pract Published Online First: 18 April 2016. doi: 10.1177/0884533616639126.

20 Johnson TW, Spurlock A, Pierce L. Survey study assessing attitudes and experiences of pediatric registered dietitians regarding blended food by gastrostomy tube feeding. Nutr Clin Pract 2015;30:402-5. 